\title{
Arztbewertungen durch valide Qualitätsdaten ergänzen
}

\author{
Der Nürnberger Juniorprofessor für Versorgungsmanagement \\ Martin Emmert sieht in Arztbewertungsportalen eine Chance \\ für mehr Transparenz in der Versorgung.
}

Wie wichtig finden Sie Arztbewertungsportale - nützen sie Patienten bei der Arztsuche?

Sie bieten eine Chance dabei, ja. Das Internet spielt generell mittlerweile eine große Rolle bei der Suche nach medizinischer Information und auch bei der Entscheidung für den richtigen Arzt. Einige Arztbewertungsportale kommen bereits unter die TOP 500 oder gar 400 der Internetseiten, die in Deutschland genutzt werden. Wir haben 2013 eine Umfrage unter 1500 online-Nutzern gemacht. Ein Drittel erklärte, von diesen Portalen schon mal gehört zu haben [1].

Online-Nutzer, das ist jener Teil der Bevölkerung, der ins Internet geht, damit ist das nicht repräsentativ für alle Bundesbürger?

Richtig. Etwa 23\% der Bevölkerung ist aktuell noch nicht online. Und wir haben bei Online-Nutzern im Vergleich zur Gesamtbevölkerung tendenziell mehr Jugendliche und einen höheren Anteil Menschen mit höherem Bildungsstatus. Was aber noch viel wichtiger ist, ist die Frage, welchen Einfluss die Portale nun auf die Nutzer haben.

\section{Und?}

Zwei Drittel der Portalnutzer haben erklärt, dass sie sich aufgrund der Bewertung auf einem Portal schon mal für einen Arzt entschieden haben. Und jeder zweite Nutzer hat sich aufgrund von Bewertungen auch schon mal gegen einen Arzt entschieden.

Können Ärzte, vor allem niedergelassene, ihr Image auf den Portalen noch ignorieren?

Ich glaube, dass es sinnvoll ist, sich mit dem Thema zu befassen. Zum einen, weil die Bedeutung der Portale noch wachsen wird und dann sehe ich auch die Chance, dass der Arzt durchaus über die Bewertungen Hinweise bekommt, was er vielleicht verbessern kann. Die Kritiken dort sind für ihn eine Art kostenloses Feedback der Patienten, er kann sehen, wie die Stimmung ist. Ob das Sprechstundenpersonal vielleicht doch nicht so freundlich ist, wie gewünscht, dass es vielleicht in der Praxis nicht so sauber ist, wie man sich das denkt und ähnliches.

\section{Bewerten Sie selber?}

Habe ich durchaus schon gemacht, ja.

Sollten Ärzte via Kommentarfunktionen auch direkt mit Bewertern und Kritikern auf den Portalen kommunizieren?

Warum nicht? Wir haben immer gefordert, dass es diese Funktion geben soll, damit Ärzte die Möglichkeit haben, direkt Stellung zu beziehen. Wenn ein Arzt dort selber kommentiert und Stellung bezieht, können andere Nutzer wiederum positiv sehen, dass er sich mit der Kritik auseinandersetzt.

\section{Soll der Arzt seine Patienten} bitten, eine Bewertung abzugeben?

Es gibt Ärzte, die das machen. Grundsätzlich ist das keine negative Sache. Wobei man dabei natürlich bedenken muss: Wenn ich Arzt wäre, würde ich natürlich die Leute ansprechen, die zufrieden aussehen.

Das gute Recht eines Arztes ...

Ja. Aber aktuell ist ein großer Nachteil an all diesen Portalen, dass das Meinungsbild überwiegend noch sehr sehr positiv ist. Daher ist der Nutzen für die User eingeschränkt. Wenn ich nach Ärzten suche, und ich finde 10 Ärzte mit der

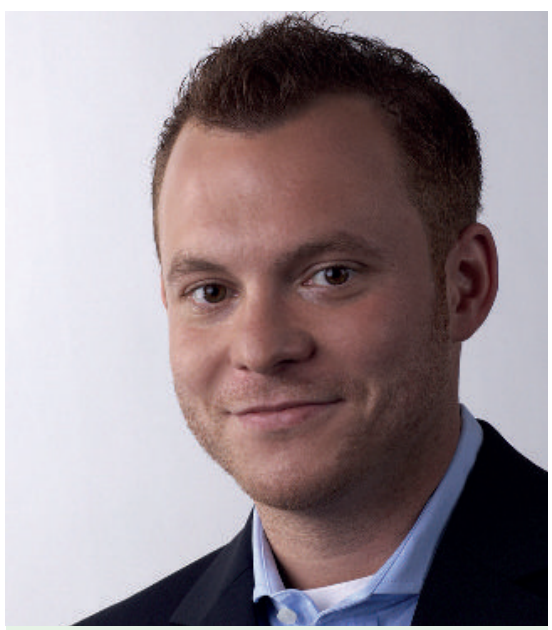

Professor Martin Emmert, Jahrgang 1979, Studium der Betriebswirtschaftslehre an der Friedrich-Alexander-Universität ErlangenNürnberg, 2008 Promotion zum Dr. rer. pol. Seit 2011 Juniorprofessor für Versorgungsmanagement an der Friedrich-Alexander-Universität Erlangen Nürnberg. Aktuell Forschungsaufenthalt an der Yale University / USA.

Note 1,0 und 2 mit der Note 1,1 und das sind alle, was mache ich dann damit? Man kann hoffen, dass es mit weiter steigendem Bekanntheitsgrad der Portale mehr kritischere Bewertungen gibt. Dass das Meinungsbild differenzierter wird.

Die Anzahl der Verteilung der Noten bleibt durch die Bank aller Untersuchungen sehr sehr positiv, ob das jetzt 78\% Ärzte mit der Note 1 bis 2 sind, oder 77 oder $81 \%$, das ist immer noch relativ identisch. Die Anzahl der Bewertungen steigt weiter, wenngleich eher langsam.

Viele Ärzte dürften eher erleichtert sein, dass die große Menge der Patienten lobt. Die Skepsis war groß, dass Ärzte im Internet vor allem beleidigt werden und dass falsche Tatsachen behauptet werden.

Das bleibt ein großes Problem. So etwas kann für einen Arzt rufschädigend, bis hin zu existenzgefährdend sein. Viele Portalbetreiber versuchen da zwar gegenzusteuern, IP-Adressenverfolgung Freitextkommentarüberprüfung und so weiter. Andererseits gibt es auch große Ambitionen, Bewertungen als Fake abzugeben, es gibt Unternehmen, die im Auftrag von Ärzten Bewertungen platzieren.

Das läuft auch in Deutschland? Ja. Und wenn es zu einer großen Zahl solcher Falschbewertungen kommt, wird das Bild verzerrt. Das ist keine Thematik, die nur auf die Arztportale zutrifft, son- 
dern auf das Internet generell. Daran müssen Nutzer immer denken.

\section{Was tun?}

Da könnte man von anderen Portalen lernen. Beim Hotelportal HRS zum Beispiel, kann man ein Hotel nur bewerten, wenn man es wirklich über HRS gebucht hat. Erst dann gibt es vom Portal eine E-Mail, über die man eine Bewertung abgeben kann.

Übertragen auf Arztportale hieße das, der Arzt müsste nach dem Besuch des Patienten diesem eine E-Mail senden, dass der ihn nun bewerten darf?

So könnte das laufen. Alternativ könnten auch die Krankenkassen diese Aufgabe übernehmen.

Die Weisse Liste fordert eine Anmeldung mit der Versichertennummer - so kann man womöglich schon etliche Falschbewertungen ausschließen?

Richtig. Solche Hürden haben allerdings offenkundig den Nachteil, dass weniger Bewertungen abgegeben werden. Die Weisse Liste, hat sich das ursprünglich einfacher vorgestellt. Aktuell schaltet man dort erst frei, wenn für einen Arzt 5 Bewertungen abgegeben wurden. Vor Zeiten sollte die Freigabe erst bei 50 Bewertungen erfolgen, dann waren es 10 , mittlerweile sind es 5, wohl auch, weil man den Aufwand unterschätzt hat. Dieses Portal hat nach wie vor zu wenige Bewertungen.

Sodass ein Nutzer dort zu sehr sehr vielen Ärzten gar keine Infos findet.

Richtig, und das hat dann Folgen. Denn wenn ich als Nutzer auf ein Portal gehe, das keine Ärzte zu meiner Region freigeschaltet hat, dann gehe ich natürlich zu einem anderen, wo ich Bewertungen finde - auch weil dort vielleicht bereits ab der ersten Bewertung freigeschaltet wird.

Auch in den USA sieht man, dass die Portale, die kaum Hürden vor einer Bewertung haben, im Bekanntheitsgrad deutlich vorne liegen. Und damit auch mehr Einfluss haben, weil man in der Online-Welt eben anonym und schnell unterwegs sein will.

Wer bewertet auf solchen Portalen?

Wir sehen, dass sich überdurchschnittlich oft Frauen auf den Portalen umsehen vielleicht lässt sich das damit erklären, dass die Frau oft die Gesundheitsmanagerin der Familie ist. Sie sucht den Arzt für alle aus. Männer wiederum bewerten tendenziell eher als Frauen.

2013 haben Sie bei einer Literaturübersicht gerade mal 23 Studien zum Thema Arztbewertungsportale weltweit gefunden, 8 davon aus Deutschland [2]. Sehr wenig. Ja, aber man muss sehen, dass dieses Thema eigentlich erst gerade mal seit 6-7 Jahren diskutiert wird.

Wie viele Arztbewertungsportale gibt es in Deutschland? In unserer letzten Erhebung kommen wir auf 24.

\section{Und weltweit?}

Das ist schwer zu sagen. In den USA würde ich 30 bis 40 schätzen. In UK sind es weniger als 10 . In den USA und vor allem in UK werden viele Krankenhäuser und Ärzte auch über öffentlich-rechtliche Systeme bewertet. In den USA gibt es das Hospital Consumer Assessment of Healthcare Providers and Systems (HCAHPS) als ein nationales Umfragesystem zu jedem Krankenhaus, hier werden landesweit Patienten befragt (siehe Link 1 am Ende, Anm. Red.). Dort werden übrigens immer erst dann die Ergebnisse freigeschaltet, wenn mindestens 100 Befragungsergebnisse zu einer Klinik vorliegen.

\section{Das ist kein Arztbewertungs- portal?}

Nein, da wird ein festgelegter, validierter Fragenkatalog auf verschiedene Wegen eingesetzt.

Womöglich erfreuen sich die überwiegend kommerziell betriebenen Arztbewertungsportale hierzulande gerade deswegen auch regen Zulaufs, weil es sonst für die Qualitätsbewertung von niedergelassenen Ärzten nichts gibt. Es könnte ja auch der Staat machen mit einer soliden jährliche Erfassung à la HCAHPS?

Ein Nebeneinander von solchen Systemen wäre das Beste. Ich finde es wünschenswert, wenn auch Behörden und Selbstverwaltungen solche Portale aufbauen würden. Ob das irgendwann mal in den Bereich des Instituts für Qualitätssicherung und Transparenz im Gesundheitswesen (IQTIG) fallen kann, das werden wir sehen. 2013 haben Sie für das Portal jameda den dortigen Datensatz für das Jahr 2012 ausgewertet [3]. Was haben Sie gefunden?

Wir haben von jameda den Datensatz für Forschungszwecke bekommen, aber keinerlei finanzielle Zuwendungen. Es waren an die 107000 Bewertungen auf jameda in dem Jahr, für insgesamt 53500 Ärzte. Damit wurden dort in dem Jahr 37\% aller Ärzte in Deutschland mindestens einmal bewertet.

Nach Fachgruppen sortiert wurden die Orthopäden mit 60\% aller Praxen am häufigsten bewertet, gefolgt von Dermatologen und Frauenärzten ( $\odot$ Abb. 3 im vorangegangenen Bericht).

Insgesamt lobte zwei Drittel der Bewerter ihre Ärzte. Dabei gibt es Unterschiede nach Facharztgruppen. Orthopäden erhielten, wie auch in einigen anderen Studien, relativ viele negative Bewertungen, ihre „Durchschnittsnote“ betrug bei jameda auch im letzten Jahr nur 2,24. Allgemeinärzte kamen auf 1,92 ( $\odot$ Abb. 2 im vorangegangenen Bericht).

Die Note 2,24, das ist ja immer noch nicht schlecht. Sehen Sie belastbare Gründe für diese Unterschiede zwischen Arztgruppen?

Die Gründe wissen wir nicht. Ich halte es für denkbar, dass es auch an den Patientenvorstellungen liegt. Man geht recht oft etwa wegen Knieschmerzen zum Orthopäden, und womöglich sind viele Patienten dann nicht zufrieden, Tenor: Mein Knie tut immer noch weh.

Sind die Verzeichnisse der Ärzte in den Portalen überhaupt vollständig?

Wir haben 2014 zuletzt Daten vorgestellt [4], nach denen in der Tat manche Portale Lücken in ihren Verzeichnissen haben. Ich nehme an, dass es immer noch Unterschiede gibt. Das ist eine Fehlerquelle, die die Portale schließen müssen.

Stichwort Freitexte. jameda hat sie, die Weisse Liste hat sie nicht, bietet damit erst gar keine Gelegenheit, Unsinn zu schreiben. Wofür sind Sie?

Ich bin für die Freitexte. Die vorrangige Frage ist, wie wir die Verbreitung und Nutzung der Portale steigern können. Auf Amazon, auf ebay... überall sind Freitexte ein beliebtes und bekanntes online-Bewertungstool. Die Nutzer wollen das.

Die Portale können vor allem die Zufriedenheit der Patienten abfragen. Die bewerten dabei über- 
wiegend die Soft Skills eines Arztes, seine Kommunikationsfähigkeit, Freundlichkeit, vielleicht auch die Sauberkeit in seiner Praxis. Sagt das irgendwas aus über die medizinischfachliche Qualität?

Auch das wissen wir nicht, wir haben keine Daten, ob die Angaben zur online erhobenen Patientenzufriedenheit am Ende mit der medizinischen Qualität eines Arztes korrelieren. Im Krankenhausbereich gibt es Hinweise darauf, dass die Noten für manche Abteilungen oder gar Ärzte mit medizinischer Qualität korrelieren, wenngleich sehr schwach.

Dabei haben wir für den Krankenhausbereich ja die externe Qualitätssicherung nach §137 SGB V, da werden medizinische Parameter erfasst, die Kliniken zum Teil auch alle 2 Jahre veröffentlichen müssen. Im niedergelassenen Bereich hätten Sie gar nicht die Daten, um zu vergleichen, ob medizinische Qualitätsparameter mit der Patientenzufriedenheit korrelieren?

Doch, diese Daten gibt es bereits vereinzelt, nur werden sie bislang nicht veröffentlicht.

\section{Welche Daten meinen Sie?}

Es gibt erste derartige Informationen, zum Beispiel aus den Selektivverträgen, den IV-Verträgen (IV: integrierte Versorgung), den Disease-Management-Programmen (DMPs). Die Daten liegen aktuell unter Verschluss bei den Kassen und Leistungserbringern und werden bislang nicht veröffentlicht.

\section{Ein Beispiel?}

Wir arbeiten mit dem Nürnberger Gesundheitsnetz QuE - Qualität und Effizienz eng zusammen. Dort weiß man intern sehr genau, wie viel Prozent der Diabetiker in den letzten 12 Monaten bei welchem Arzt eine Routineuntersuchung hatten bzw. wie viel Prozent der Patienten richtig eingestellt sind. Ich halte das für das A\&O, dass solche Daten auch veröffentlicht werden, parallel zu den Werten der Patientenzufriedenheit.

Man kann durchaus von Patienten, die in DMPs eingeschrieben sind, Qualitätsindikatoren erfassen und auswerten. Auch die großen Kassen machen das längst, manche Krankenkassen wissen exakt, wer von den Ärzten wie bei den DMPs abschneidet. Prinzipiell wären die Daten, zumindest für alle Praxen in Deutschland, die an solchen Selektivverträgen teilnehmen. vorhanden. Meine Forderung ist, die Informationen aus DMPs, aus Hausarztmodellen, etc... aufzubereiten und dann auch zu veröffentlichen.

\section{Praxis für Praxis?}

Ja. Damit könnte der Informationsgehalt auch bei Portalen für die Arztsuche deutlich aussagekräftiger werden. Dass ich bei den Portalen auch lesen kann, wo ist denn der Orthopäde gut, ist der gut am Knie, am Rücken, an der Hand, was auch immer.

Ist man da in den USA weiter?

Nein, für niedergelassene Ärzte nicht. Wohl aber in Großbritannien. Dort können Sie Punkt für Punkt im Internet nachlesen, wie viele Ärzte in eigener Praxis gewisse Qualitätsparameter bei der Behandlung von Diabetes-Patienten oder bei der chirurgischen Versorgung erfüllen. Dort werden die Daten im Rahmen des "Quality and Outcomes-Framework“ ermittelt und dann auch veröffentlicht (siehe Link 2, Anm. Red.).

Wie sehen Sie die Rankings, die meisten Portale sortieren die Ärzte einer Suchliste nach ihrem Abschneiden? Ist das Ranking wirklich sinnvoll?

Was man mit so einem Portal rüber bringt, ist ja eigentlich immer ein Ranking bzw. eine Empfehlung. Das ist die Intention. Und die Leute wollen ein Ranking, sie wollen wissen, wer gut oder schlecht ist. Auch die Studien, die wir haben, zeigen, dass ein Ranking hilfreich ist bei der Information, die die User suchen, und deswegen finde ich, dass es sinnvoll ist.

Die Portale erstellen die Rankings oft nach einem Mix, bei dem die Noten, aber auch die Zahl an Bewertungen eingeht.

Korrekt. Das ist auch richtig - wenn ein Arzt 20 Bewertungen hat mit der Durchschnittsnote 1,1, dann hat diese Note meines Erachtens ein höheres Gewicht als die Bewertung eines anderen Arztes mit der Note 1,0 , hinter der aber nur eine einzige Bewertung steckt. Denn bei letzterem ist natürlich das Risiko größer, dass das Gesamtabschneiden an einer einzigen und womöglich gar gefälschten Bewertung hängt. Es ist richtig, dass ein gewisses System dort einen Ausgleich schafft. Ich persönlich bin nicht genau im Bilde darüber, wie das Bewertungssystem ausschaut. Man kann das nur in gewissen Grenzen nachlesen. Da wünsche ich mir mehr Transparenz.

\section{Veröffentlichen?}

Ja. Für die Nachvollziehbarkeit wäre es besser, diese Algorithmen würden veröffentlicht. Manche Firmen mögen es als Geschäftsgeheimnis auffassen, aber ich wäre dafür, dass diese Algorithmen veröffentlicht werden.

Werden die Portale die Qualität der Versorgung verbessern?

Ganz spannendes Thema. Wir denken, dass Ärzte durch die Portale im positiven Sinne motiviert werden können, bessere Bewertungen zu erreichen und deswegen versuchen, sich zu verbessern, indem sie die Anregungen der Patienten aufgreifen. Ich bin sicher, dass die Portale mehr Transparenz und Anreize zu Qualitätsverbesserungen in das System bringen können. Daten dazu, gibt es noch keine. Wir wollen demnächst untersuchen, wie Ärzte die Portale nutzen.

\section{Literatur}

1 Emmert M, Meier F, Pirsch F et al. Physician Choice Making and Characteristics Associated With Using Physician-Rating Websites: Cross-Sectional Study. J Med Internet Res 2013; 15: e187

2 Emmert M, Sander U, Pisch F. Eight questions about physician-rating websites: a systematic review. J Med Internet Res. 2013; 15: e24

3 Emmert M, Meier F. An analysis of online evaluations on a physician rating website: evidence from a German public reporting instrument. J Med Internet Res 2013; 15: e157

4 Emmert M, Gerstner B, Sander U et al. Eine Bestandsaufnahme von Bewertungen auf Arztbewertungsportalen am Beispiel des Nürnberger Gesundheitsnetzes Qualität und Effizienz (QuE). Gesundheitsökonomie \& Qualitätsmanagement 2014; 19: 161-167

\section{Das Interview führte Bernhard Epping}

Weitere Informationen
Link 1: http://www.hcahpsonline.
org/home.aspx
Link 2: http://www.england.nhs.uk/
resources/resources-for-ccgs/out-
frwrk/, http://qof.hscic.gov.uk/
(Stand der Links: 16.07.2015)

